\title{
THE COEFFICIENTS OF UNIVALENT FUNCTIONS
}

\author{
M. S. ROBERTSON
}

1. Introduction. Let the function

$$
f(z)=c_{0}+c_{1} z+c_{2} z^{2}+\cdots+c_{n} z^{n}+\cdots, \quad c_{n} \text { real, }
$$

be regular and convex in the direction of the imaginary axis for $|z|<1$. Thus each circle $|z|=r, 0<r<1$, is mapped by $f(z)$ into a contour $C_{r}$ which has the property that straight lines parallel to the imaginary axis cut $C_{r}$ in at most two points. Since the coefficients are all real, $C_{r}$ is symmetric about the real axis. For

$$
f\left(r e^{i \theta}\right)=U(r, \theta)+i V(r, \theta)
$$

we have $\partial U(r, \theta) / \partial \theta \leqq 0$ for $0<\theta<\pi$. In other words, $z f^{\prime}(z)$ is typically real for $|z|<1$. It is well known $[1,2]^{1}$ that the coefficients $c_{n}$ are bounded, $\left|c_{n}\right| \leqq\left|c_{1}\right|, n=1,2, \cdots$, and [3] have the representation

$$
c_{n}=\frac{c_{1}}{n \pi} \int_{0}^{\pi} \frac{\sin n \theta}{\sin \theta} d \alpha(\theta)
$$

where $\alpha(\theta)$ is a nondecreasing function of $\theta$ in $(0, \pi)$ normalized so that

$$
\frac{1}{\pi} \int_{0}^{\pi} d \alpha(\theta)=1
$$

A sufficient condition that $f(z)$, given by the series (1.1), be regular and convex in the direction of the imaginary axis for $|z|<1$ is that the sequence $\left\{c_{n}\right\}$ be monotonic of order 4 , a theorem due to L. Fejér [4]. A sequence $\left\{c_{n}\right\}$ is said to be monotonic of order $p$ if each of the differences

$$
\Delta^{(k)} c_{n}=c_{n}-C_{k, 1} c_{n+1}+C_{k, 2} c_{n+2}-\cdots+(-1)^{k} C_{k, k} c_{n+k}
$$

are non-negative for $k=0,1,2, \cdots, p ; n=0,1,2, \cdots$. This suffciency test implies, among other inequalities, that $0 \leqq c_{n}-c_{n+1}$. This suggests the problem of finding an upper bound for $c_{n}-c_{n+1}$ for functions $f(z)$ given by (1.1) which are convex in the direction of the imaginary axis for $|z|<1$. The example $c_{1} z(1+z)^{-1}$ shows that the upper bound $2\left|c_{1}\right|$ is sharp. However, if we consider the differences $c_{n-1}-c_{n+1}$ we obtain an inequality which is not so immediately obvious. This inequality is stated in the following theorem.

Presented to the Society, September 17, 1945; received by the editors May 21, 1945.

${ }^{1}$ Numbers in brackets refer to the references cited at the end of the paper. 
Theorem A. Let the function

$$
f(z)=z+c_{2} z^{2}+\cdots+c_{n} z^{n}+\cdots
$$

be regular and convex in the direction of the imaginary axis for $|z|<1$ and be real on the real axis. Then the coefficients $c_{n}$ satisfy the inequalities

$$
\begin{array}{rr}
c_{n-1}-c_{n+1} \leqq 4 n\left(n^{2}-1\right)^{-1}\left(1-\left|c_{n}\right|\right), & n=2,4,6, \cdots ; c_{1}=1 ; \\
\left|c_{n-1}-c_{n+1}\right| \leqq 4 n\left(n^{2}-1\right)^{-1}\left(1-c_{n}\right), & n=3,5,7, \cdots .
\end{array}
$$

The factor $4 n\left(n^{2}-1\right)^{-1}$ cannot be replaced by a smaller one and the equality signs are attained for the function $z(1-z)^{-1}$.

2. Some trigonometric inequalities. For the proof of Theorem $A$ we need the trigonometric inequality to follow.

LEMMA 1. If $n$ is a positive integer then for all real values of $\theta$

$$
\begin{aligned}
(n+1) \frac{\sin (n-1) \theta}{\sin \theta}-(n-1) \frac{\sin (n+1) \theta}{\sin \theta} & \\
& \leqq 4\left(n-\frac{\sin n \theta}{\sin \theta}\right) .
\end{aligned}
$$

Proof. We consider first the case where $n$ is an odd positive integer.

$$
\begin{aligned}
8 \sum_{k=1}^{(n-1) / 2} & {\left[\cos \frac{n \theta}{2}-\cos (2 k-1) \frac{\theta}{2}\right]^{2} } \\
= & (4 n-4) \cos ^{2} \frac{n \theta}{2}-16 \cos \frac{n \theta}{2} \cdot \sum_{1}^{(n-1) / 2} \cos (2 k-1) \frac{\theta}{2} \\
& +8 \sum_{1}^{(n-1) / 2} \cos ^{2}(2 k-1) \frac{\theta}{2} \\
= & (4 n-4) \cos ^{2} \frac{n \theta}{2}-8 \cos \frac{n \theta}{2} \cdot \frac{\sin ((n-1) \theta / 2)}{\sin (\theta / 2)} \\
& +8\left[\frac{n-1}{4}+\frac{\sin (n-1) \theta}{4 \sin \theta}\right] \\
= & (2 n-2)(1+\cos n \theta)-4\left[\frac{\sin ((2 n-1) \theta / 2)}{\sin (\theta / 2)}-1\right] \\
& +2 n-2+2 \frac{\sin (n-1) \theta}{\sin \theta}
\end{aligned}
$$




$$
\begin{aligned}
= & 4 n+(2 n-2) \cos n \theta+4\left[\frac{\sin (n-1) \theta}{\sin \theta}-\frac{\sin ((2 n-1) \theta / 2)}{\sin (\theta / 2)}\right] \\
& -2 \frac{\sin (n-1) \theta}{\sin \theta} \\
= & 4 n+(2 n-2) \cos n \theta-4 \frac{\sin n \theta}{\sin \theta}-2 \frac{\sin (n-1) \theta}{\sin \theta} \\
= & 4\left(n-\frac{\sin n \theta}{\sin \theta}\right)-(n+1) \frac{\sin (n-1) \theta}{\sin \theta}+(n-1) \frac{\sin (n+1) \theta}{\sin \theta} .
\end{aligned}
$$

Again, if $n$ is an even integer we have

$$
\begin{aligned}
4(1- & \left.\cos \frac{n \theta}{2}\right)^{2}+8 \sum_{k=1}^{(n-2) / 2}\left[\cos \frac{n \theta}{2}-\cos k \theta\right]^{2} \\
= & \left(4-8 \cos \frac{n \theta}{2}+4 \cos ^{2} \frac{n \theta}{2}\right)+(4 n-8) \cos ^{2} \frac{n \theta}{2} \\
& -16 \cos \frac{n \theta}{2} \sum_{1}^{(n-2) / 2} \cos k \theta+8 \sum_{1}^{(n-2) / 2} \cos ^{2} k \theta \\
= & 4-8 \cos \frac{n \theta}{2}+2(1+\cos n \theta)+(2 n-4)(1+\cos n \theta) \\
& -16 \cos \frac{n \theta}{2}\left[-\frac{1}{2}+\frac{\sin ((n-1) \theta / 2)}{2 \sin (\theta / 2)}\right] \\
& +8\left[\frac{n-3}{4}+\frac{\sin (n-1) \theta}{4 \sin \theta}\right] \\
= & 4 n-4+(2 n-2) \cos n \theta-8 \cos \frac{n \theta}{2} \cdot \frac{\sin ((n-1) \theta / 2)}{\sin (\theta / 2)} \\
& +2 \frac{\sin (n-1) \theta}{\sin \theta} \\
= & 4\left(n-\frac{\sin n \theta}{\sin \theta}\right)-(n+1) \frac{\sin (n-1) \theta}{\sin \theta}+(n-1) \frac{\sin (n+1) \theta}{\sin \theta}
\end{aligned}
$$

Hence, in either case we have, as required,

$$
\begin{aligned}
4\left(n-\frac{\sin n \theta}{\sin \theta}\right)+ & (n-1) \frac{\sin (n+1) \theta}{\sin \theta} \\
& -(n+1) \frac{\sin (n-1) \theta}{\sin \theta} \geqq 0 .
\end{aligned}
$$


Lemma 2. If $n$ is a positive integer then for all real values of $\theta$

$$
\begin{aligned}
4\left[1+\frac{\sin 2 \theta}{\sin \theta}+\frac{\sin 3 \theta}{\sin \theta}+\cdots+\right. & \left.\frac{\sin (n-1) \theta}{\sin \theta}\right] \\
& -(n-2) \frac{\sin n \theta}{\sin \theta} \leqq n^{2} .
\end{aligned}
$$

Lemma 2 follows easily from the identity

$$
\frac{\sin n \theta}{\sin \theta}+2 \sum_{k=1}^{n-1} \frac{\sin k \theta}{\sin \theta}=\left(\frac{\sin (n \theta / 2)}{\sin (\theta / 2)}\right)^{2}
$$

and the inequality $A_{n}(0) \geqq 0$ where

$$
A_{n}(\theta) \equiv n^{2}+n \frac{\sin n \theta}{\sin \theta}-2\left(\frac{\sin (n \theta / 2)}{\sin (\theta / 2)}\right)^{2} .
$$

To prove the inequality $A_{n}(\theta) \geqq 0$ we observe first that $A_{1}(\theta)=A_{2}(\theta)$ $\equiv 0$. We prove next that $A_{n+1}(\theta) \geqq A_{n-1}(\theta)$ whence $A_{n}(\theta) \geqq 0$ by induction.

$$
\begin{aligned}
A_{n+1}(\theta)-A_{n-1}(\theta) & \\
= & +(n+1) \frac{\sin (n+1) \theta}{\sin \theta}-(n-1) \frac{\sin (n-1) \theta}{\sin \theta} \\
- & 2\left[\left(\frac{\sin ((n+1) \theta / 2)}{\sin (\theta / 2)}\right)^{2}-\left(\frac{\sin ((n-1) \theta / 2)}{\sin (\theta / 2)}\right)^{2}\right] \\
= & 4 n+(n-1)\left[\frac{\sin (n+1) \theta-\sin (n-1) \theta}{\sin \theta}\right] \\
& +2 \frac{\sin (n+1) \theta}{\sin \theta}-4 \frac{\sin n \theta}{\sin \theta}(1+\cos \theta) \\
= & 4\left(n-\frac{\sin n \theta}{\sin \theta}\right)+(n-1) \frac{\sin (n+1) \theta}{\sin \theta} \\
& -(n+1) \frac{\sin (n-1) \theta}{\sin \theta} \geqq 0
\end{aligned}
$$

by Lemma 1 .

3. Proof of Theorem A. From (2.1) we have

$$
\frac{1}{(n-1) \pi} \int_{0}^{\pi} \frac{\sin (n-1) \theta}{\sin \theta} d \alpha(c)
$$




$$
\begin{aligned}
& -\frac{1}{(n+1) \pi} \int_{0}^{\pi} \frac{\sin (n+1) \theta}{\sin \theta} d \alpha(\theta) \\
& \leqq \frac{4 n}{n^{2}-1}\left[\frac{1}{\pi} \int_{0}^{\pi} d \alpha(\theta)-\frac{1}{n \pi} \int_{0}^{\pi} \frac{\sin n \theta}{\sin \theta} d \alpha(\theta)\right] .
\end{aligned}
$$

From (1.2) and (3.1) we have immediately

$$
c_{n-1}-c_{n+1} \leqq \frac{4 n}{n^{2}-1}\left(1-c_{n}\right), \quad n=2,3, \cdots .
$$

Since

$$
-f(-z)=z-c_{2} z^{2}+c_{3} z^{3}-c_{4} z^{4} \cdots
$$

is also regular and convex in the direction of the imaginary axis for $|z|<1$ it follows that (3.2) may be replaced by the inequalities given in Theorem A.

The function

$$
f(z)=\sum_{1}^{\infty} \frac{1}{n} \frac{\sin n \theta}{\sin \theta} z^{n}
$$

is regular and convex in the direction of the imaginary axis for $|z|<1$ since $z f^{\prime}(z)=z\left(1-2 z \cos \theta+z^{2}\right)^{-1}$ is typically real, indeed univalent and star-like for $|z|<1$. For this function

$$
\begin{aligned}
\lim _{\theta \rightarrow 0} \frac{c_{n-1}-c_{n+1}}{1-c_{n}} \\
\quad=\lim _{\theta \rightarrow 0}\left(\frac{n}{n^{2}-1}\right) \frac{(n+1) \sin (n-1) \theta-(n-1) \sin (n+1) \theta}{n \sin \theta-\sin n \theta} \\
\quad=4 n \cdot\left(n^{2}-1\right)^{-1} .
\end{aligned}
$$

As a consequence of Theorem A we easily obtain the following theorem.

THEOREM B. Let the function

$$
F(z)=z+a_{2} z^{2}+\cdots+a_{n} z^{n}+\cdots
$$

have real coefficients and be regular and univalent for $|z|<1$. Then

$$
\begin{aligned}
(n+1) a_{n-1}-(n-1) a_{n+1} & \leqq 4\left(n-\left|a_{n}\right|\right), n=2,4,6, \cdots, \\
\left|(n+1) a_{n-1}-(n-1) a_{n+1}\right| & \leqq 4\left(n-a_{n}\right), \quad n=3,5,7, \cdots .
\end{aligned}
$$

The equality signs are attained by the function $z \cdot(1-z)^{-2}$ which is univalent in the unit circle. 
4. Some additional inequalities for the coefficients. From the inequality (2.3) by an argument similar to that used in the proof of Theorems A and B we obtain the following theorem.

THEOREM C. With the same hypothesis as in Theorem $\mathrm{B}$, the following inequalities for the coefficients of $F(z)$ hold:

$$
4\left(1+a_{2}+a_{3}+\cdots+a_{n-1}\right)-(n-2) a_{n} \leqq n^{2} .
$$

In a somewhat similar way by using the trigonometric inequality

$$
\begin{aligned}
& \left(n^{2}-1\right)(n+2)\left(2-\frac{\sin 2 \theta}{\sin \theta}\right)-2(n-1)(n+2) \frac{\sin (n+1) \theta}{\sin \theta} \\
& \quad+(n+3)(n+2) \frac{\sin n \theta}{\sin \theta}+\left(n^{2}-3 n-2\right) \frac{\sin (n+2) \theta}{\sin \theta} \geqq 0
\end{aligned}
$$

we may obtain another set of inequalities for the coefficients of $f(z)$ of Theorem A :

(4.3) $2\left(n^{2}-1\right)\left(1-c_{2}-c_{n+1}\right)+\left(n^{2}+3 n\right) c_{n}+\left(n^{2}-3 n-2\right) c_{n+2} \geqq 0$.

We omit the details of the proof.

\section{REFERENCES}

1. M. S. Robertson, On the theory of univalent functions, Ann. of Math. vol. 37 (1936) pp. 374-408.

2. - Analytic functions star-like in one direction, Amer. J. Math. vol. 58 (1936) pp. 465-472.

3. - On the coefficients of a typically-real function, Bull. Amer. Math. Soc. vol. 41 (1935) pp. 565-572.

4. L. Fejer, Trigonometrische Reihen und Potenzreihen mit mehrfach monotoner Koeffizientenfolge, Trans. Amer. Math. Soc. vol. 39 (1936) pp. 18-59.

RUTGERS UNIVERSITY 